EXTENDED REPORT

\title{
Effects of ibuprofen on molecular markers of cartilage and synovium turnover in patients with knee osteoarthritis
}

\author{
E Gineyts, J A Mo, A Ko, D B Henriksen, S P Curtis, B J Gertz, P Garnero, P D Delmas
}

Ann Rheum Dis 2004;63:857-861. doi: 10.1136/ard.2003.007302

See end of article for authors' affiliations

.......................

Correspondence to: Dr E Gineyts, Research Unit 403, Hôpital Edouard Herriot, Pavillon F, Place d'Arsonval, 69003 Lyon, France; gineyts@lyon. inserm.fr

Accepted 24 August 2003
Objective: The aim of this study was to evaluate the effect of ibuprofen on the urinary excretion of Cterminal crosslinking telopeptide of type II collagen (CTX-II) and urinary glucosyl galactosyl pyridinoline (Glc-Gal-PYD), two new molecular markers of cartilage and synovial tissue metabolism, respectively, in patients with knee osteoarthritis (OA).

Methods: We studied 201 patients with knee pain and radiographic evidence of knee OA who were on treatment with non-steroidal anti-inflammatory drugs (NSAIDs) prior to study initiation. After an initial screening visit, patients were withdrawn from their pre-study NSAID and, following a flare of their OA symptoms, were randomised to ibuprofen $(2400 \mathrm{mg} /$ day) or placebo. Urinary CTX-II and Glc-Gal-PYD levels were measured at time of randomisation (baseline) and after 4-6 weeks of treatment.

Results: After 4 to 6 weeks, urinary CTX-II (+17\%, $p=0.023)$ and Glc-Gal-PYD $(+10 \%, p=0.020)$ increased significantly from baseline in the placebo group whereas marginal or no increase was observed in the ibuprofen group (CTX-II $+2 \%$, NS and Glc-Gal-PYD $+4 \%, p=0.045$ ). For urinary CTX-II, the difference in the change from baseline between placebo and ibuprofen treated groups was significant (13\%, $p=0.017)$. At baseline, urinary levels of CTX-II and Glc-Gal-PYD were higher in patients with knee swelling $(n=127)$ than in those without $(n=74)(p<0.02$ for both markers). When patients were stratified according to presence or absence of knee swelling at baseline, the increases over 4-6 weeks of urinary CTX-II and Glc-Gal-PYD in the placebo group were restricted to patients with knee swelling $1+22 \%$ from baseline, $p=0.001$ and $+12 \%, p=0.011$, for urinary CTX-II and Glc-Gal-PYD respectively). In patients with knee swelling who were treated with ibuprofen this increase was not observed and the difference from placebo was significant for urinary CTX-II $(p=0.014)$.

Conclusion: In patients with a flare of knee OA, specifically in patients with evidence of joint inflammation documented by knee swelling, there was a significant increase in markers reflecting cartilage and synovium metabolism that could partly be prevented by high doses of ibuprofen. These data suggest that patients with a flare of knee OA are characterised by increased cartilage and synovial tissue degradation, which may be partly prevented by high doses of NSAIDs.
O steoarthritis (OA) is a chronic disease, and the most common of the joint disorders. It is characterised by degraded articular cartilage and concomitant alteration of synovium and subchondral bone metabolism, leading to pain and stiffness in joints and to impaired physical function. ${ }^{1}$

Currently there are very few structural modifying drugs for OA treatment, and most commonly used medications are symptom modifying drugs, such as non-steroidal antiinflammatory drugs (NSAIDs)..$^{2-4}$ These treatments are believed to alleviate only pain, and their effects on cartilage and synovium metabolism in patients with $\mathrm{OA}$ are still debated. For instance, in vitro studies suggest that NSAIDs may inhibit or enhance the synthesis of cartilage proteoglycans. ${ }^{5-6}$ Data from in vivo studies using several models of OA suggest that NSAID administration may accelerate articular damage. ${ }^{7-9}$ Conversely, other in vitro and in vivo studies have shown that some NSAIDs exert a favourable effect on the overall metabolism of cartilage in OA. ${ }^{10-13}$

To assess the potential benefit of treatment in modifying joint structure, plain $x$ rays of the joints are usually used. However, because of the limited precision of this technique, long term studies of 2-3 years are necessary to assess efficacy of such treatments. ${ }^{14}$

Molecular markers released into biological fluids that reflect joint tissue metabolism have been proposed as a possible alternative method for assessment of disease progression. ${ }^{15}{ }^{16}$ Because of their rapid and dynamic changes, they may be useful for monitoring treatment effects on joint tissue metabolism. Recently, two newly developed specific and sensitive biochemical markers reflecting the turnover of cartilage and synovial tissue matrices, respectively, have been developed. An immunoassay using antibodies recognising type II collagen C-telopeptide fragments in urine (CTX-II) has been developed to monitor type II collagen degradation of cartilage. ${ }^{17}$ Urinary excretion of a glycosylated analogue of pyridinoline, glucosyl galactosyl pyridinoline (Glc-Gal-PYD), measured by high performance liquid chromatography, has been shown to reflect specifically the degradation of synovial tissue. $^{18}$ Recent cross-sectional studies have shown that urinary CTX-II and Glc-Gal-PYD are increased in patients with OA. ${ }^{17-19}$ A cross-sectional evaluation of a panel of biochemical markers of bone, cartilage, and synovium metabolism in patients with knee OA showed that these two markers were the most predictive factors of the current extent of joint damage evaluated by $x$ rays. ${ }^{19}$ In patients with rheumatoid arthritis, urinary excretion of CTX-II and of GlcGal-PYD are also increased and predicted subsequent joint destruction assessed by $x$ rays of the hands and feet. ${ }^{20}{ }^{21}$ In addition, a small study in patients with Paget's disease has

Abbreviations: ARA, American Rheumatism Association; CTX-II, type II collagen; Glc-Gal-PYD, glucosyl galactosyl pyridinoline; NSAID, nonsteroidal anti-inflammatory drug; $\mathrm{OA}$, osteoarthritis 
shown that bisphosphonate zoledronate, which has been suggested to have chondroprotective effects in animals, ${ }^{22}$ decreases urinary CTX-II within days of treatment, suggesting that such molecular markers may indeed respond to therapy. ${ }^{23}$

The aim of this placebo controlled prospective study was to analyse the effect of a non-selective NSAID, ibuprofen, on urinary excretion of CTX-II and Glc-Gal-PYD in patients with knee OA.

\section{PATIENTS AND METHODS Patients}

The local ethics committee approved the study, and informed consent was obtained from all patients. The study comprised 201 patients with clinical and radiographic evidence of knee OA, who fulfilled the American Rheumatism Association (ARA) criteria for functional classes I, II, or III (table 1). The protocol inclusion criteria were explicit to include patients with a clinical diagnosis of OA of the knee (tibiofemoral compartment) or hip for greater than 6 months based on clinical and radiographic criteria. For all patients with OA of the hip, criteria were: $(a)$ pain in the affected hip on motion or weight bearing for the majority of days during the previous month, at least partially relieved by rest; and $(b)$ joint space narrowing as assessed by the reader at each individual study site. For patients with OA of the knee, criteria were $(a)$ pain in the affected knee on motion or weight bearing for the majority of the days during the previous month, at least partially relieved by rest, as above; and (b) tibiofemoral osteophytes and tibiofemoral joint space narrowing as assessed by the reader at each individual study site. These are standard inclusion criteria used in NSAID OA studies.

These patients were a subset of a larger randomised, multicentre, double blind study comparing the efficacy of rofecoxib, a COX-2 selective inhibitor, and ibuprofen. ${ }^{24}$ All screened patients were previously on a non-selective NSAID. After an initial screening visit to ensure study eligibility, patients were withdrawn from the pre-study NSAID and, following a flare of their OA symptoms, were randomised to ibuprofen $2400 \mathrm{mg}$ /day (800 mg three time daily) or placebo. The definition of OA "flare" was pre-specified in the protocol as follows. Prior to randomisation and following discontinuation of NSAIDs during the washout period specified for the individual pre-study NSAID the patient was taking (defined as a period of time of at least five half lives for the individual NSAID in question), the patient had to satisfy the following three flare criteria at the randomisation visit prior to being randomised: (a) a minimum of $40 \mathrm{~mm}$ on a $100 \mathrm{~mm}$ visual analogue scale (VAS) of patient reported pain when walking on a flat surface; $(b)$ an increase of $15 \mathrm{~mm}$ on a VAS of patient reported pain when walking on a flat surface (both WOMAC $^{\mathrm{TM}}$ section A, question 1) compared with pre-study baseline recorded at screening visit; and (c) worsening of disease status of at least 1 point on a 5 point scale compared with the screening visit recording as assessed by the investigator.

For the classification of patients into the two groups according to the presence or absence of knee joint swelling at baseline, the study investigators were responsible for examining the study joint for swelling. Soft tissue swelling was considered as present if there was palpable or visible evidence of capsular distension thought to be due to either synovial thickening and/or joint effusion. Bone swelling, nodule formation, and joint deformity was excluded from consideration. Swelling was graded as absent (0) or present (1).

For each patient, urine samples were obtained at randomisation (baseline) and after 4 and 6 weeks of treatment, or at the time of discontinuation if the patient did not complete the study (15\% of patients). CTX-II and Glc-Gal-PYD were

\begin{tabular}{|c|c|c|}
\hline Baseline patient characteristics & $\begin{array}{l}\text { Placebo } \\
(n=45)\end{array}$ & $\begin{array}{l}\text { Ibuprofen }(2400 \mathrm{mg}) \\
(\mathrm{n}=156)\end{array}$ \\
\hline \multicolumn{3}{|l|}{ Gender } \\
\hline Female & 37 (82\%) & 117 (75\%) \\
\hline Male & $8(18 \%)$ & $39(25 \%)$ \\
\hline Age, years & $62(10)$ & $61(10)$ \\
\hline \multicolumn{3}{|l|}{ ARA functional class } \\
\hline 1 & $8(18 \%)$ & $18(12 \%)$ \\
\hline ॥ & $25(55 \%)$ & $97(62 \%)$ \\
\hline III & $12(27 \%)$ & $41(26 \%)$ \\
\hline \multicolumn{3}{|l|}{ Knee swelling } \\
\hline Yes & $36(80 \%)$ & $91(58 \%)$ \\
\hline No & $9(20 \%)$ & $65(42 \%)$ \\
\hline
\end{tabular}

determined from these urine samples, which were collected in the morning at the time of the clinic visit.

\section{Molecular markers of cartilage and synovium turnover}

Urinary CTX-II was measured with a specific competitive ELISA using a mouse monoclonal antibody directed against a linear six amino acid epitope (EKGPDP) of the human type II collagen $\mathrm{C}$ telopeptide. ${ }^{17}$ The intra- and inter-assay coefficients of variation were less than 8 and $10 \%$ respectively. ${ }^{17}$

Urinary Glc-Gal-PYD was measured by high performance liquid chromatography according to a modification of a previously published technique. ${ }^{18}$ Intra- and inter-assay variations were less than 8 and $11 \%$, respectively. After extraction of the cross-links by cellulose CFl partition column chromatography, they were eluted from a reversed phase HPLC on a Beckman ultrasphere ODS ( $5 \mu \mathrm{m}$, $25 \mathrm{~cm} \times 4.6 \mathrm{~mm}$ ) protected by a Brownlee RP-18 Guard cartridge $(7 \mu \mathrm{m}, 15 \times 3 \mathrm{~mm})$ at a flow rate of $1 \mathrm{ml} / \mathrm{min}$ with an isocratic elution of $12 \%$ acetonitrile in $0.15 \%$ of HBFA. The eluent was quantified by fluorimetry by monitoring for fluorescence at an emission wavelength of $395 \mathrm{~nm}$ and an excitation wavelength of $297 \mathrm{~nm}$. To standardise the HPLC assay, PYD purified from bone was used instead of Glc-GalPYD as it is difficult to obtain large amounts of Glc-Gal-PYD from urine. ${ }^{18}$ The PYD standard, which elutes a few minutes later than Glc-Gal-PYD on the chromatographic profile, was calibrated against purified human urine Glc-Gal-PYD, the structure of which was confirmed by mass spectrometry. ${ }^{18}$ Concentrations for each marker in urine were expressed per urinary creatinine concentration assessed by routine laboratory methods.

\section{Statistical analyses}

Because urinary levels of cartilage and synovium markers were not normally distributed, all data were log transformed to obtain normal distribution before statistical analysis. Differences in marker levels between groups were assessed by Student's $t$ test using the standard error from an analysis of covariance model with terms for treatment and baseline covariate. Only samples from patients who had both a baseline sample and at least one sample while on treatment were included in the analysis. For patients with more than one on treatment sample, the median of the on treatment values were used. These included 44 patients with only one on treatment sample, 156 patients with two on treatment samples and 1 patient with three on treatment samples.

\section{RESULTS}

At baseline, urinary levels of CTX-II and Glc-Gal-PYD did not differ significantly $(p>0.05)$ between the ibuprofen and 
placebo groups (table 2). In patients on placebo there was a significant increase in urinary CTX-II and Glc-Gal-PYD during follow up, with an average increase of $17 \%$ $(\mathrm{p}=0.023 \quad v$ baseline $)$ and $10 \% \quad(\mathrm{p}=0.020 \quad v$ baseline $)$, respectively (table 2 ). In patients treated with ibuprofen, no significant increase in urinary CTX-II was observed $(\mathrm{p}>0.05$ vs baseline), whereas Glc-Gal-PYD increased slightly (4\%, $\mathrm{p}=0.045$ ) (table 2). After 4 to 6 weeks of treatment, the increase in urinary CTX-II from baseline was significantly higher in the placebo than the ibuprofen group $(+13 \%$, $\mathrm{p}=0.017$ ). For Glc-Gal-PYD, the difference between groups did not reach statistical significance $(p>0.05)$.

When patients were divided into two groups according to the presence or absence of knee swelling at baseline $(n=127$ with knee swelling, $\mathrm{n}=74$ without), urinary levels of CTX-II and Glc-Gal-PYD were significantly elevated in those with knee swelling (table 3). In patients on placebo, the increase in urinary CTX-II and Glc-Gal-PYD during the study was primarily seen in those presenting knee swelling $(+22 \%$, $\mathrm{p}=0.001$ and $+12 \%, \mathrm{p}=0.011$, for CTX-II and Glc-Gal-PYD respectively). No significant change in patients without knee swelling (table 3) was observed. In patients with knee swelling this increase was not observed in the ibuprofen treatment group (table 3 ) and the difference from the placebo group was significant for urinary CTX-II $(p=0.014)$. Baseline levels of urinary Glc-Gal-PYD and CTX-II were only partially correlated $\left(\mathrm{r}^{2}=0.17, \mathrm{p}<0.001\right)$.

\section{DISCUSSION}

We found that patients with a flare of knee OA manifesting clinically detectable knee swelling were characterised by increased urinary excretion of markers reflecting cartilage and synovium degradation respectively. The level of these two markers increased over 4-6 weeks on placebo, and the increase was partly prevented by high dose ibuprofen. CTX-II and Glc-Gal-PYD are specific degradation products of cartilage and synovial tissue, respectively, and although the relation between such molecular markers and joint tissue metabolism is still unclear, these data suggest that high doses of ibuprofen may possibly have on effect on cartilage and

Table 2 Markers of cartilage and synovium turnover in patients with knee OA treated with ibuprofen or placebo

\begin{tabular}{|c|c|c|}
\hline Treatment group & $\begin{array}{l}\text { Placebo } \\
(n=45)\end{array}$ & $\begin{array}{l}\text { Ibuprofen } \\
(\mathrm{n}=156)\end{array}$ \\
\hline \multicolumn{3}{|l|}{ Cartilage marker CTX-II (ng/mmol Cr) } \\
\hline Baseline, mean (SD in log scale) & $226(0.63)$ & $225(0.77)$ \\
\hline $\begin{array}{l}\text { Week 4-6, mean (SD in log } \\
\text { scale) }\end{array}$ & $265(0.72)$ & $229(0.72)$ \\
\hline $\begin{array}{l}\text { Intra-treatment ratio, mean } \\
(95 \% \mathrm{Cl}) \S\end{array}$ & $\begin{array}{l}1.17^{*} \\
(1.06 \text { to } 1.30)\end{array}$ & $\begin{array}{l}1.02 \\
(0.96 \text { to } 1.08)\end{array}$ \\
\hline $\begin{array}{l}\text { Inter-treatment ratio, mean } \\
(95 \% \mathrm{Cl})\end{array}$ & $\begin{array}{l}0.87 \\
10.77 \text { to } 0.971\end{array}$ & \\
\hline$p$ value for treatment effect & 0.017 & \\
\hline \multicolumn{3}{|c|}{ Synovium marker: Glc-Gal-PYD (nmol/mmol $\mathrm{Cr}$ ) } \\
\hline Baseline, mean (SD in log scale) & $5.7(0.36)$ & $6.0(0.40)$ \\
\hline $\begin{array}{l}\text { Week } 4-6 \text {, mean (SD in log } \\
\text { scale) }\end{array}$ & $6.3(0.34)$ & $6.2(0.40)$ \\
\hline $\begin{array}{l}\text { Intra-treatment ratio, mean } \\
(95 \% \mathrm{Cl}) \S\end{array}$ & $\begin{array}{l}1.10^{\dagger} \\
(1.02 \text { to } 1.18)\end{array}$ & $\begin{array}{l}1.04^{\ddagger} \\
(1.00 \text { to } 1.09)\end{array}$ \\
\hline Inter-treatment ratio, mean & 0.95 & \\
\hline$(95 \% \mathrm{Cl})$ & (0.87 to 1.04$)$ & \\
\hline $\mathrm{p}$ value for treatment effect & NS & \\
\hline
\end{tabular}

Means are geometric means.

Paired test $v$ baseline: ${ }^{*} \mathrm{p}=0.023 ; \mathrm{t}=0.020 ; \neq \mathrm{p}=0.045$.

$\S$ Estimates and $p$ values are from a one way multiple analysis of covariance model with treatment as a factor and $\log _{\text {(baseline) }}$ as a covariate. synovium metabolism in patients with active knee OA, especially those with clinical evidence of synovitis.

NSAIDs are commonly used to treat OA because of their analgesic and anti-inflammatory effects, and have been shown to be effective in improving pain and disability. ${ }^{2-4}$ Daily administration of $2400 \mathrm{mg}$ ibuprofen is effective in treating symptoms of pain and problems with mobility in OA as assessed by standardised clinical scores. ${ }^{24}$ However, these clinical indices are poorly related to changes in joint structure, and it remains unknown whether NSAIDs could also affect joint tissue metabolism.

In this study, we selected two newly developed markers that specifically reflect cartilage degradation and synovium metabolism. The basic hypothesis is that such markers could reflect the metabolic processes in joint tissues and could be used for the diagnosis, prognosis, and monitoring of joint disease. However, the relation between marker concentrations in blood or urine and the metabolic processes in the joint tissues may be altered by various factors such as the clearance from the joint compartment, which may be affected by the inflammation process. We cannot exclude that the effects we observed are related in part to changes in clearance. However, in vitro studies using human joint tissue explants have shown that CTX-II and Glc-Gal-PYD are released specifically by cartilage and synovial tissue, respectively. ${ }^{17}{ }^{18}$ We recently showed in vivo that increased urinary excretion of CTX-II and Glc-Gal-PYD is associated with more rapid progression of joint destruction in patients with early rheumatoid arthritis and in patients with knee OA, suggesting that these markers do in fact relate to joint damage. ${ }^{19-21}$ The ability of these two new markers to monitor the efficiency of treatment in OA has not yet been investigated.

In patients on placebo we found that during the 4-6 week study duration, urinary CTX-II and Glc-Gal-PYD significantly increased, mainly in those patients presenting with clinical signs of synovitis-that is, with knee swelling and effusion. These data suggest that joint inflammation is associated with increased synovial activity and cartilage breakdown, which can be detected in vivo by these specific markers. These findings are in agreement with recent data showing that knee OA patients with inflammation of the synovium, as evaluated directly by joint arthroscopy, had a greater progression of cartilage damage over 1 year, as assessed by arthroscopy. ${ }^{25}$ Thus, synovitis is likely to play an important role in the process of joint damage in OA. A specific non-invasive marker of that process, such as Glc-Gal-PYD, is likely be of significant clinical value in detecting OA patients at increased risk for progression of joint damage, as recently suggested by data in patients with rheumatoid arthritis. ${ }^{20}$

In patients treated with high dose ibuprofen, increase in urinary CTX-II was lower than that observed in the placebo group during the same period. However, after ibuprofen treatment the levels of both markers remained higher than those in patients without synovial effusions, suggesting that treatment did not completely prevent synovial activity and cartilage destruction. Nevertheless, our study suggests that ibuprofen may have some effects on joint tissue metabolism, in agreement with other studies showing that NSAIDs reduce cartilage breakdown in animal models of OA. ${ }^{10} 12$ 26-28

Our study had some limitations. The lower number of patients in the placebo group as predefined in the original trial may have decreased the power to detect significant difference with the active treatment group. The study lasted only 4-6 weeks and thus we could not investigate long term effects of ibuprofen on these new biochemical markers. We did not assess joint damage by $x$ rays or MRI and thus could not relate the changes in urinary CTX-II and Glc-Gal-PYD directly to changes in joint structure. Extended longitudinal studies with measurement of biochemical and imaging 
Table 3 Markers of cartilage and synovium turnover in OA patients with and without joint swelling in ibuprofen and placebo groups

\begin{tabular}{|c|c|c|c|c|c|c|}
\hline \multirow[b]{2}{*}{ Joint swelling } & \multicolumn{2}{|l|}{ Placebo } & \multicolumn{2}{|l|}{ Ibuprofen } & \multicolumn{2}{|l|}{ Overall } \\
\hline & Yes $(n=36)$ & No $(n=9)$ & Yes $(n=91)$ & No $(n=65)$ & Yes $(n=127)$ & No $(n=74)$ \\
\hline \multicolumn{7}{|l|}{ Cartilage marker: CTX-\|I (ng/mmol Cr) } \\
\hline Baseline, mean (SD in log scale) & $245(0.58)$ & $164(0.73)$ & $251 \S(0.82)$ & $194(0.67)$ & $249 \ddagger(0.76)$ & $190(0.67)$ \\
\hline Week 4-6, mean (SD in log scale) & $295(0.65)$ & $174(0.87)$ & $253(0.76)$ & $199(0.63)$ & $265(0.73)$ & $196(0.66)$ \\
\hline Intra-treatment ratio, mean & 1.22 & 1.01 & 1.03 & 1.00 & 1.06 & 1.03 \\
\hline$(95 \% \mathrm{Cl}) \S$ & (1.09 to 1.37$)$ & $(0.80$ to 1.27$)$ & $(0.96$ to 1.11$)$ & $(0.92$ to 1.09$)$ & (1.00 to 1.13$)$ & (0.95 to 1.12$)$ \\
\hline $\begin{array}{l}\text { Inter-treatment ratio, mean } \\
(95 \% \mathrm{Cl})+t\end{array}$ & $\begin{array}{l}0.84 \\
(0.74 \text { to } 0.97)\end{array}$ & & & & & \\
\hline p value for treatment effect & 0.014 & & & & & \\
\hline \multicolumn{7}{|c|}{ Synovium marker: Glc-Gal-PYD $(\mathrm{nmol} / \mathrm{mmol} \mathrm{Cr})$} \\
\hline Baseline, mean (SD in log scale) & $5.9(0.35)$ & $4.9(0.37)$ & $6.4^{* *}(0.42)$ & $5.5(0.36)$ & $6.2 \cdot(0.41)$ & $5.4(0.36)$ \\
\hline Week 4-6, mean (SD in log scale) & $6.6(0.31)$ & $5.2(0.44)$ & $6.4(0.45)$ & $5.9(0.30)$ & $6.5(0.42)$ & $5.8(0.32)$ \\
\hline Intra-treatment ratio, mean (95\% CI) & $1.12^{+}$ & 1.01 & 1.03 & 1.06 & 1.04 & 1.08 \\
\hline & (1.03 to 1.22$)$ & $(0.85$ to 1.20$)$ & (0.98 to 1.09$)$ & (0.99 to 1.13$)$ & (0.99 to 1.09$)$ & (1.01 to 1.15$)$ \\
\hline Inter-treatment ratio, mean & 0.92 & & & & & \\
\hline $\begin{array}{l}(95 \% \mathrm{Cl}) \mathrm{t \dagger} \\
\mathrm{p} \text { value for treatment effect }\end{array}$ & (0.83 to 1.02$)$ & & & & & \\
\hline$p$ value for treatment effect & NS & & & & & \\
\hline
\end{tabular}

Means are geometric means.

Paired test $v$ baseline: ${ }^{*} p=0.001 ;+p=0.011$

Unpaired test $v$ patients without joint swelling: $\ddagger p=0.011 ; \S p=0.038 ; \uparrow p=0.015 ;{ }^{* *} p=0.025$.

t†One way multiple analysis of covariance model with treatment, presence of joint swelling, and treatment $\times$ joint swelling as factors and log(baseline) as a covariate.

markers will be necessary to confirm the potential chondroprotective properties of this NSAID.

In summary, we found that patients with inflammatory knee OA are characterised by increased turnover of synovium and cartilage. Ibuprofen treatment, which is known to effectively reduce the signs and symptoms of OA, appeared to reduce this turnover as monitored by the urinary markers CTX-II and Glc-Gal-PYD. Whether ibuprofen can also prevent cartilage loss and reduce synovium degradation in patients with OA remains to be investigated.

\section{Authors' affiliations}

E Gineyts, P Garnero, P D Delmas, INSERM Research Unit 403, Lyon, France

J A Mo, D B Henriksen, Nordic Bioscience, Herlev, Denmark

A Ko, S P Curtis, B J Gertz, Merck, Rahway, NJ, USA

$P$ Garnero, Synarc, Lyon, France

\section{REFERENCES}

1 Felson DT. Osteoarthritis: new insights. Part 1: The disease and its risk factors. Ann Intern Med 2000;133:635-46.

2 Hochberg MC, Altman RD, Brandt KD, Clark BM, Dieppe PA, Griffin M, et al. Guidelines for the medical management of osteoarthritis. Part I: Osteoarthritis of the hip. Arthritis Rheum 1995;38:1535-40.

3 Hochberg MC, Altman RD, Brandt KD, Clark BM, Dieppe PA, Griffin MR, et al. Guidelines for the medical management of osteoarthritis. Part II: Osteoarthritis of the knee. Arthritis Rheum 1995;38:1541-6.

4 Baum C, Kennedy DL, Forbes MB. Utilization of nonsteroidal antiinflammatory drugs. Arthritis Rheum 1985;28:686-92.

5 Rainsford KD. Mechanisms of NSAIDs on joint destruction in osteoarthritis. Agents Actions Suppl 1993;44:39-43.

6 Dingle JT. The effect of nonsteroidal antiinflammatory drugs on human articular cartilage glycosaminoglycan synthesis. Osteoarthritis Cartilage 1999;7:313-14.

7 Brandt KD. Effects of nonsteroidal anti-inflammatory drugs on chondrocyte metabolism in vitro and in vivo. Am J Med 1987;83:29-34.

8 Serni U, Mannoni A, Benucci $M$. Is there preliminary in vivo evidence for an influence of nonsteroidal antiinflammatory drugs on progression in osteoartritis? Part II: evidence from animal models. Osteoarthritis Cartilage 1999;7:351-2.

9 Gencosmanoglu BE, Eryavuz M, Dervisoglu S. Effects of some nonsteroidal anti-inflammatory drugs on articular cartilage of rats in an experimental model of osteoarthritis. Res Exp Med 2001;200:215-26.

10 Fernandes JC, Caron JP, Martel-Pelletier J, Jovanovic D, Mineau F, Tardif G, et al. Effects of tenidap on the progression of osteoarthritic lesions in a canine experimental model. Suppression of metalloprotease and interleukin-1 activity. Arthritis Rheum 1997;40:284-94.
11 Blot L, Marcelis A, Devogrlaer JP, Manicourt DH. Effect of diclofenac, aceclofenac and meloxicam on the metabolism of proteoglycans and hyaluronan in osteaoarthritic human cartilage. Br J Pharmacol 2000;131:1413-21.

12 Hwa SY, Burkhardt D, Little C, Ghosh PJ. The effects of orally administered diacerein on cartilage and subchondral bone in an ovine model of osteoarthritis. J Rheumatol 2001;28:825-34.

13 Dooley M, Spencer CM, Dunn CJ. Aceclofenac: a reappraisal of its use in the management of pain and rheumatic disease. Drugs 2001;61:1351-78.

14 Ravaud P, Giraudeau B, Auleley GR, Drape JL, Rousselin B, Paolozzi L, et al. Variability in knee radiographing: implication for definition of radiological progression in medial knee osteoarthritis. Ann Rheum Dis 1998;57:624-9.

15 Garnero P, Rousseau JC, Delmas PD. Molecular basis and clinical use of biochemical markers of bone, cartilage, and synovium in joint diseases. Arthritis Rheum 2000;43:953-68.

16 Vignon E, Garnero P, Delmas PD, Avouac B, Bettica P, Boers M, et al. Recommendations for the registration of drugs used in the treatment of osteoarthritis: an update on biochemical markers. Osteoarthritis Cartilage 2001;9:289-93.

17 Christgau S, Garnero P, Fledelius C, Jensen T, Moniz M, Ensig M, et al. Collagen type II C-telopeptide fragments as an index of cartilage degradation. Bone 2001;29:209-15.

18 Gineyts E, Garnero P, Delmas PD. Urinary excretion of glucosyl galactosyl pyridinoline: a specific biochemical marker of synovium degradation. Rheumatology 2001;40:315-21.

19 Garnero P, Piperno M, Gineyts E, Christau S, Delmas PD, Vignon E. Cross sectional evaluation of biochemical markers of bone, cartilage, and synovial tissue metabolism in patients with knee osteoarthritis: relations with disease activity and joint damage. Ann Rheum Dis 2001;60:619-26.

20 Garnero P, Gineyts E, Christgau S, Finck B, Delmas PD. Association of baseline levels of urinary glucosyl-galactosyl pyridinoline and type II collagen C-telopeptide with progression of joint destruction in patients with early rheumatoid arthritis. Arthritis Rheum 2002;46:21-30.

21 Garnero P, Landewé R, Boers M, Verhoeven A, van der Linden S, Christgau S, et al. Association of baseline levels of markers of bone and cartilage degradation with long-term progression of joint damage in patients with early rheumatoid arthritis. Arthritis Rheum 2002;46:2847-56.

22 Podworny NV, Kandel RA, Renlund RC, Grynpas MD. Partial chondroprotective effect of zoledronate in a rabbit model of inflammatory arthritis. J Rheumatol 1999;26:1972-82.

23 Garnero P, Christgau S, Delmas PD. The bisphosphonate zoledronate decreases type Il collagen breakdown in patients with Paget's disease of bone. Bone 2001;28:461-4

24 Saag K, Van der Heijde D, Fisher C, Samara A, DeTora L, Bolognese J, et al. Rofecoxib, a new cyclooxygenase 2 inhibitor, shows sustained efficacy, comparable with other nonsteroidal anti-inflammatory drugs: a 6-week and a 1-year trial in patients with osteoarthritis. Arch Fam Med 2000;9:1124-34.

25 Ayral X, Pickering EH, Woodworth TG, Mackillop N, Dougados M. Synovitis predicts the arthroscopic progression of medial tibiofemoral knee osteoarthritis. ACR 65th San Francisco, CA: Scientific Meeting, 2001.

26 Ratcliffe A, Azzo W, Saed-Nejad F, Lane N, Rosenwasser MP, Mow VC. In vivo effects of naproxen on composition, proteoglycan metabolism, and matrix metalloproteinase activities in canine articular cartilage. J Orthop Res 1993;11:163-71. 
27 Fernandes JC, Martel-Pelletier J, Jovanovic D, Tardif G, DiBattista JA Lascau-Coman $\mathrm{V}$, et al. The effects of tenidap on canine experimental osteoarthritis: II. Study of the expression of collagenase-1 and interleukin 1 beta by in situ hybridization. J Rheumatol 1998;25:

951-8.
28 Kosaka N, Ishiwa J, Sato T, Nagase H, Ito A. Rhein, an active metabolite of diacerein, down-regulates the production of pro-matrix metalloproteinases-1, $-3,-9$ and -13 and up-regulates the production of tissue inhibitor of metalloproteinase- 1 in cultured rabbit articular chondrocytes. Osteoarthritis Cartilage 2001 ; 9:257-63.

\section{$\mathrm{ECHO}$}

\section{Intra-articular steroid for knee osteoarthritis}

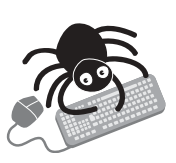

Please visit the Annals of the Rheumatic Diseases website [www. annrheumdis. com] for a link to the full text of this article.
A vailable evidence suggests that intra-articular steroid injection for knee osteoarthritis usually gives relief for perhaps one to four weeks although longer benefit has been reported. There is concern though, that repeated injections might damage the joint. Workers in New Zealand have carried out a meta-analysis of randomised, placebo controlled trials.

Although ten studies satisfied the inclusion criteria the main analysis was confined to six of them. With a total of 160 patients in the treatment groups and 157 in control groups the relative risk of improvement in symptoms up to two weeks after steroid injection was 1.66 (95\% confidence interval 1.37-2.01). The number needed to treat to achieve one improvement varied between 1.3 and 3.5. Two studies were considered to be of high quality. Neither study alone provided significant evidence of improvement at 16-24 weeks but pooling of data (63 treated patients, 61 controls) did show significant improvement at that time (relative risk 2.09 ( 1.20 to 3.65), number needed to treat 4.4). Doses (prednisone equivalent) varied from $6.25 \mathrm{mg}$ to $80 \mathrm{mg}$. The authors of the review comment that a triamcinolone dose of $20 \mathrm{mg}$ (equivalent to $25 \mathrm{mg}$ of prednisone) seems to be efficacious for pain control at two weeks. Only one study included measurements of joint space. In this study repeated injections of triamcinolone $40 \mathrm{mg}$ were used and there was no significant loss of joint space at two years compared with controls. The two studies using high doses reported longer benefit. One study led to a recommendation that joint lavage should be added to steroid injection when knee effusion persists after one or two steroid injections.

Steroid injections produce symptomatic improvement for up to two weeks and possibly for 16-24 weeks though a higher dose (equivalent to $50 \mathrm{mg}$ of prednisone) may be needed to achieve the longer term improvement.

A BMJ 2004;328:869-870 